\title{
Inequalities on weighted classical pythagorean means, Tracy-Singh products, and Khatri-Rao products for hermitian operators
}

\author{
Arnon Ploymukda, Pattrawut Chansangiam* \\ Department of Mathematics, Faculty of Science, King Mongkut's Institute of Technology Ladkrabang, Bangkok 10520, Thailand \\ * Corresponding author: pattrawut.ch@kmitl.ac.th
}

Article history

Received 18 December 2018

Revised 10 September 2019

Accepted 26 September 2019

Published Online 15 April 2020

\begin{abstract}
We establish a number of operator inequalities between three kinds of means, namely, weighted arithmetic/harmonic/geometric means, and two kinds of operator products, namely, Tracy-Singh products and Khatri-Rao products. In this study, we have validated the data under certain assumptions relying on (opposite) synchronization, comparability, and spectra of operators. The tensor product of operators, and Tracy-Singh/Khatri-Rao products of matrices as special cases are presented.
\end{abstract}

Keywords: weighted arithmetic mean, weighted geometric mean, weighted harmonic mean, TracySingh product, Khatri-Rao product

@ 2020 Penerbit UTM Press. All rights reserved

\section{INTRODUCTION}

Throughout this paper, $\mathrm{H}$ and $\mathrm{K}$ denote the complex separable Hilbert spaces. When $\mathrm{X}$ and $\mathrm{Y}$ are Hilbert spaces, let $\mathrm{B}(\mathrm{X}, \mathrm{Y})$ be the algebra of all bounded linear operators from $\mathrm{X}$ into $\mathrm{Y}$, and abbreviate $\mathrm{B}(\mathrm{X}, \mathrm{X})$ to $\mathrm{B}(\mathrm{X})$. Denoted by $\mathrm{B}(\mathrm{H})^{+}$the cone of positive operators on $\mathrm{H}$. For any Hermitian operators $A$ and $B$ on $\mathrm{H}$, the partial order $A \ddot{O} B$ indicates that $A-B \in \mathrm{B}(\mathrm{H})^{+}$. Two Hermitian operators $A$ and $B$ are comparable if $A \ddot{\mathrm{O}} B$ or $A \tilde{\mathrm{N}} B$. The expressions $A>0$ and $A \in \mathrm{B}(\mathrm{H})^{++}$have the same meaning so that $A$ is both positive and invertible.

This paper focuses on the three classical Pythagorean means, namely, the arithmetic mean, the geometric mean, and the harmonic mean. Over the years, theory of these kinds of means for matrices and operators are significantly developed, see e.g. [1-3] and references therein. Recall that for any $t \in[0,1]$, the $t$-weighted arithmetic mean of $A, B \in \mathrm{B}(\mathrm{H})$ is defined by

$$
A "{ }_{t} B=(1-t) A+t B \text {. }
$$

The $t$-weighted harmonic mean of $A, B \in \mathrm{B}(\mathrm{H})^{++}$is defined by

$$
A !_{t} B=\left[(1-t) A^{-1}+t B^{-1}\right]^{-1} .
$$

In general, for any $A, B \in \mathrm{B}(\mathrm{H})^{+}$, we define

$$
A !_{t} B=\lim _{\varepsilon \rightarrow 0^{+}}(A+\varepsilon I) !_{t}(B+\varepsilon I) \text {. }
$$

Here, the limit is taken in the strong-operator topology. The $t$ weighted geometric mean of two operators $A, B \in \mathrm{B}(\mathrm{H})^{+}$is defined by

$$
A \#_{t} B=A^{1 / 2}\left(A^{-1 / 2} B A^{-1 / 2}\right)^{t} A^{1 / 2} \text {. }
$$

For arbitrary positive operators, we define their weighted geometric mean by using the continuity argument as that for (1). In brief, we write $A " B, A ! B$ and $A \# B$ for $A{ }_{1 / 2} B, A !_{1 / 2} B$ and $A \#_{1 / 2} B$, respectively.
Weighted classical Pythagorean means have the following remarkable properties where for any $A, B \in \mathrm{B}(\mathrm{H})^{+}, \quad X \in \mathrm{B}(\mathrm{H})$, and $t \in[0,1]$, we have

$$
\begin{aligned}
& A \sigma_{t} B=B \sigma_{1-t} A, \\
& X^{*}\left(A \sigma_{t} B\right) X \tilde{\mathrm{N}}\left(X^{*} A X\right) \sigma_{t}\left(X^{*} B X\right),
\end{aligned}
$$

It is well-known that for any $A_{1}, B_{1}, A_{2}, B_{2} \in \mathrm{B}(\mathrm{H})^{+}$, we have

$$
\left(A_{1} \# B_{1}\right) \otimes\left(A_{2} \# B_{2}\right)=\left(A_{1} \otimes A_{2}\right) \#\left(B_{1} \otimes B_{2}\right) .
$$

Here, $\otimes$ denotes the tensor product. Recently, the theory of tensor product for operators is extended to that of Tracy-Singh product and Khatri-Rao product for operators, see e.g. [4-7]. The identity shown was generalized to that for weighted geometric means and Tracy-Singh products as follows:

Proposition 1 ([8]). For any $A_{1}, B_{1}, A_{2}, B_{2} \in \mathrm{B}(\mathrm{H})^{+}$and $t \in[0,1]$, we have

$\left.\left(A_{1} \#_{t} B_{1}\right)\right)\left(A_{2} \#_{t} B_{2}\right)=\left(\begin{array}{lll}\left.A_{1}\right) & \left.A_{2}\right) \#_{t}\left(B_{1}\right) & B_{2}\end{array}\right)$.

In this paper, we establish further inequalities between three kinds of weighted Pythagorean means, namely, weighted arithmetic/ harmonic/geometric means, and two kinds of operator products, namely, Tracy-Singh product and Khatri-Rao product. Our results include tensor product of operators, and Tracy-Singh/Khatri-Rao products of matrices as special cases.

The outline for the rest of paper is as follows. In Section 2, we present the preliminary results on Tracy-Singh product and Khatri-Rao product of Hilbert space operators. Section 3 begins with introducing the (opposite) synchronization between two ordered pairs of Hermitian operators. Then, we establish operator inequalities involving TracySingh products and weighted arithmetic/harmonic means under the assumptions that two pairs of operators are (opposite) synchronous. In Section 4, we prove certain operator inequalities concerning KhatriRao products and weighted arithmetic/harmonic/ geometric means under suitable assumptions about synchronization, comparability, and spectra of operators. 


\section{PRELIMINARIES ON TRACY-SINGH PRODUCT AND} KHATRI-RAO PRODUCT OF HILBERT SPACE OPERATORS

In order to define the Tracy-Singh product for Hilbert space operators, we have to fix the following orthogonal decompositions:

$$
\mathrm{H}=\bigoplus_{i=1}^{m} \mathrm{H}_{i}, \quad \mathrm{~K}=\bigoplus_{k=1}^{n} \mathrm{~K}_{k}
$$

where $\mathrm{H}_{i}$ and $\mathrm{K}_{j}$ are Hilbert spaces for $i, j$. Thus, each operator $A \in \mathrm{B}(\mathrm{H})$ and $B \in \mathrm{B}(\mathrm{K})$ can be uniquely represented as operator matrices

$$
A=\left[A_{i j}\right]_{i, j=1}^{m, m} \quad \text { and } \quad B=\left[B_{k l}\right]_{k, l=1}^{n, n}
$$

where $A_{i j} \in \mathrm{B}\left(\mathrm{H}_{j}, \mathrm{H}_{i}\right)$ and $B_{k l} \in \mathrm{B}\left(\mathrm{K}_{l}, \mathrm{~K}_{k}\right)$ for each $i, j=1, \quad, m$ and $k, l=1, \quad, n$.

Definition 2. Let $A=\left[A_{i j}\right]_{i, j=1}^{m, m}$ and $B=\left[B_{k l}\right]_{k, l=1}^{n, n}$ be operator matrices in $\mathrm{B}(\mathrm{H})$ and $\mathrm{B}(\mathrm{K})$, respectively. We define the Tracy-Singh product of $A$ and $B$ to be the bounded linear operator from $\bigoplus_{i, k=1}^{m, n} \mathrm{H}_{i} \otimes \mathrm{K}_{k}$ into itself, represented by

$$
\text { A) } B=\left[\left[A_{i j} \otimes B_{k l}\right]_{k l}\right]_{i j} \text {. }
$$

Lemma 3 ([4]). Let $A_{1}, B_{1}, A_{2}, B_{2}$ be compatible operators. Then

(i) $\left.\left.\left.\left.\left.\left(A_{1}+B_{1}\right)\right)\left(A_{2}+B_{2}\right)=A_{1}\right) A_{2}+A_{1}\right) B_{2}+B_{1}\right) A_{2}+B_{1}\right) B_{2}$.

(ii) $\left.\left.\left.\left(\alpha A_{1}\right)\right) A_{2}=A_{1}\right)\left(\alpha A_{2}\right)=\alpha\left(A_{1}\right) A_{2}\right)$ for any $\alpha \in$.

(iii) $\left.\left.\left.\left(A_{1}\right) A_{2}\right)\left(B_{1}\right) B_{2}\right)=\left(A_{1} B_{1}\right)\right)\left(A_{2} B_{2}\right)$.

(iv) If $A_{1}$ and $A_{2}$ are invertible, then $\left.\left.\left(A_{1}\right) A_{2}\right)^{-1}=A_{1}^{-1}\right) A_{2}^{-1}$.

(v) If $A_{1} \mathrm{O} A_{2} \mathrm{O} 0$ and $B_{1} \mathrm{O} B_{2} \mathrm{O} 0$, then $\left.\left.A_{1}\right) B_{1} \ddot{\mathrm{O}} A_{2}\right) B_{2} \mathrm{Ö} 0$.

To define the Khatri-Rao product of operators, we fix the decomposition (5) and assume that $m=n$.

Definition 4. Let $A=\left[A_{i j}\right]_{i, j=1}^{n, n} \in \mathrm{B}(\mathrm{H})$ and $B=\left[B_{i j}\right]_{i, j=1}^{n, n} \in \mathrm{B}(\mathrm{K})$. The Khatri-Rao product of $A$ and $B$ is defined to be the bounded linear operator from $\bigoplus_{i=1}^{n} \mathrm{H}_{i} \otimes \mathrm{K}_{i}$ into itself, represented by

$$
A^{*} B=\left[A_{i j} \otimes B_{i j}\right]_{i, j=1}^{n, n} .
$$

Lemma 5. ([5]). There exists a bounded linear operator $Z$ such that

$$
\left.A^{*} B=Z^{*}(A) B\right) Z
$$

for any $A \in \mathrm{B}(\mathrm{H})$ and $B \in \mathrm{B}(\mathrm{K})$.

The operator $Z$ in Lemma 5 is called the selection operator associated with the ordered tuple $(\mathrm{H}, \mathrm{K})$.

\section{OPERATOR INEQUALITIES ON WEIGHTED CLASSICAL PYTHAGOREAN MEANS AND TRACY-SINGH PRODUCTS}

In this section, we establish operator inequalities involving weighted arithmetic/harmonic means and Tracy-Singh products under the assumptions that two pairs of operators are (opposite) synchronous.

Definition 6. Two ordered pairs $\left(A_{1}, A_{2}\right)$ and $\left(B_{1}, B_{2}\right)$ of Hermitian operators are said to be synchronous if either

$A_{i} \tilde{\mathrm{N}} B_{i}$ for $i=1,2$, or $A_{i} \mathrm{O} B_{i}$ for $i=1,2$.

The pairs $\left(A_{1}, A_{2}\right)$ and $\left(B_{1}, B_{2}\right)$ are said to be opposite synchronous if either

$A_{1} \tilde{\mathrm{N}} B_{1}$ and $A_{2} \ddot{\mathrm{O}} B_{2}$, or $A_{1} \ddot{\mathrm{O}} B_{1}$ and $A_{2} \tilde{\mathrm{N}} B_{2}$.
Now, we establish operator inequalities involving weighted harmonic means and Tracy-Singh products.

Theorem 7. Let $A_{1}, B_{1}, A_{2}, B_{2} \in \mathrm{B}(\mathrm{H})^{+}$and $t \in[0,1]$.

(i) If $\left(A_{1}, A_{2}\right)$ and $\left(B_{1}, B_{2}\right)$ are synchronous, then

$\left.\left(A_{1} !_{t} B_{1}\right)\right)\left(A_{2} !_{t} B_{2}\right)$ Ö $\left.\left.\left(A_{1}\right) A_{2}\right) !_{t}\left(B_{1}\right) B_{2}\right)$.

(ii) If $\left(A_{1}, A_{2}\right)$ and $\left(B_{1}, B_{2}\right)$ are opposite synchronous, then

$\left.\left.\left.\left(A_{1} !_{t} B_{1}\right)\right)\left(A_{2} !_{t} B_{2}\right) \tilde{\mathrm{N}}\left(A_{1}\right) A_{2}\right) !_{t}\left(B_{1}\right) B_{2}\right)$.

Proof. (i) First, suppose $A_{1}, B_{1}, A_{2}, B_{2}>0$. The case $A_{1} \mathrm{O} B_{1}, A_{2} \mathrm{O} B_{2}$ leads to $A_{1}^{-1} \tilde{\mathrm{N}} B_{1}^{-1}$ and $A_{2}^{-1}, B_{2}^{-1}$. Thus, by Lemma 3 we have

$$
\left.\left(A_{1}^{-1}-B_{1}^{-1}\right)\right)\left(A_{2}^{-1}-B_{2}^{-1}\right) \text { Ö } 0 \text {. }
$$

The case $A_{1} \tilde{\mathrm{N}} B_{1}, A_{2} \tilde{\mathrm{N}} B_{2}$ also leads to the inequality (12). It follows from (12) that

$$
\begin{aligned}
0 \tilde{\mathrm{N}} & \left.\left.\left.t(1-t) A_{1}^{-1}\right) A_{2}^{-1}+t(1-t) B_{1}^{-1}\right) B_{2}^{-1}-t(1-t) A_{1}^{-1}\right) B_{2}^{-1} \\
& \left.-t(1-t) B_{1}^{-1}\right) A_{2}^{-1} \\
= & {\left.\left.\left[(1-t)-(1-t)^{2}\right] A_{1}^{-1}\right) A_{2}^{-1}+\left(t-t^{2}\right) B_{1}^{-1}\right) B_{2}^{-1} } \\
& \left.\left.-t(1-t) A_{1}^{-1}\right) B_{2}^{-1}-t(1-t) B_{1}^{-1}\right) A_{2}^{-1} \\
= & \left.\left.(1-t) A_{1}^{-1}\right) A_{2}^{-1}+t B_{1}^{-1}\right) B_{2}^{-1} \\
& \left.-\left[(1-t) A_{1}^{-1}+t B_{1}^{-1}\right]\right)\left[(1-t) A_{2}^{-1}+t B_{2}^{-1}\right] .
\end{aligned}
$$

Thus

$$
\begin{aligned}
& \left.\left[(1-t) A_{1}^{-1}+t B_{1}^{-1}\right]\right)\left[(1-t) A_{2}^{-1}+t B_{2}^{-1}\right] \\
& \left.\left.\tilde{\mathrm{N}}(1-t) A_{1}^{-1}\right) A_{2}^{-1}+t B_{1}^{-1}\right) B_{2}^{-1} .
\end{aligned}
$$

Hence

$\left.\left.\left(A_{1}\right) A_{2}\right) !_{t}\left(B_{1}\right) B_{2}\right)$

$$
\begin{aligned}
& \left.\left.=\left\{(1-t)\left(A_{1}\right) A_{2}\right)^{-1}+t\left(B_{1}\right) B_{2}\right)^{-1}\right\}^{-1} \\
& \left.\left.=\left\{(1-t) A_{1}^{-1}\right) A_{2}^{-1}+t B_{1}^{-1}\right) B_{2}^{-1}\right\}^{-1} \\
& \left.\tilde{\mathrm{N}}\left\{\left[(1-t) A_{1}^{-1}+t B_{1}^{-1}\right]\right)\left[(1-t) A_{2}^{-1}+t B_{2}^{-1}\right]\right\}^{-1} \\
& \left.=\left[(1-t) A_{1}^{-1}+t B_{1}^{-1}\right]^{-1}\right)\left[(1-t) A_{2}^{-1}+t B_{2}^{-1}\right]^{-1} \\
& \left.=\left(A_{1} ! B_{1}\right)\right)\left(A_{2} !_{t} B_{2}\right) .
\end{aligned}
$$

For arbitrary $A_{1}, B_{1}, A_{2}, B_{2} \mathrm{O} 0$, perturb each of them with $\varepsilon I$ and then take limit as $\varepsilon \rightarrow 0^{+}$.

(ii) By continuity, we may assume that $A_{1}, B_{1}, A_{2}, B_{2}>0$. If $\left(A_{1}, A_{2}\right)$ and $\left(B_{1}, B_{2}\right)$ are opposite synchronous, then we get the reverse of (12). Hence, in this case, we get (11).

Corollary 8. If two positive operators $A$ and $B$ are comparable, then for any $t \in[0,1]$,

$$
\begin{array}{ll}
\left.\left(A !_{t} B\right)\right) & \left.\left.\left(A !_{t} B\right) \text { Ö }(A) \quad A\right) !_{t}(B) B\right), \\
\left.\left(A !_{t} B\right)\right) & \left.\left.\left(B !_{t} A\right) \text { N }(A) B\right) !_{t}(B) A\right), \\
\left.\left(A !_{t} B\right)\right) & \left.\left.\left(B^{-1} !_{t} A^{-1}\right) \ddot{\mathrm{O}}(A) B^{-1}\right) !_{t}(B) A^{-1}\right), \\
\left.\left(A !_{t} B\right)\right) & \left.\left.\left(A^{-1} !_{t} B^{-1}\right) \tilde{\mathrm{N}}(A) A^{-1}\right) !_{t}(B) B^{-1}\right) .
\end{array}
$$

Here, in (15) and (16), we assume further that $A$ and $B$ are invertible.

Proof. First, suppose $A \tilde{\mathrm{N}} B$. Then the pairs $(A, A)$ and $(B, B)$ are synchronous. By Theorem 7, we get (13). Since $(A, B)$ and $(B, A)$ are opposite synchronous, Theorem 7 yields the inequality (14). The synchronization between $\left(A, B^{-1}\right)$ and $\left(B, A^{-1}\right)$ implies the inequality (15). The opposite synchronization between $\left(A, A^{-1}\right)$ and $\left(B, B^{-1}\right)$ implies the inequality (16). The case $A \ddot{O} B$ can be similarly treated. 
Next, we discuss relations between weighted arithmetic means and Tracy-Singh products in terms of inequalities.

Theorem 9. Let $A_{1}, B_{1}, A_{2}, B_{2} \in \mathrm{B}(\mathrm{H})$ be Hermitian operators and $t \in[0,1]$

(i) If $\left(A_{1}, A_{2}\right)$ and $\left(B_{1}, B_{2}\right)$ are synchronous, then

$$
\left.\left.\left.\left(A_{1} "{ }_{t} B_{1}\right)\right)\left(A_{2}{ }_{t} B_{2}\right) \tilde{\mathrm{N}}\left(A_{1}\right) A_{2}\right){ }^{\prime \prime}{ }_{t}\left(B_{1}\right) B_{2}\right) \text {. }
$$

(ii) If $\left(A_{1}, A_{2}\right)$ and $\left(B_{1}, B_{2}\right)$ are opposite synchronous, the

$$
\left.\left.\left.\left(A_{1}{ }^{\prime}{ }_{t} B_{1}\right)\right)\left(A_{2}{ }^{\prime}{ }_{t} B_{2}\right) \text { Ö }\left(A_{1}\right) A_{2}\right){ }^{\prime \prime}{ }_{t}\left(B_{1}\right) B_{2}\right) \text {. }
$$

Proof. (i) Since $\left(A_{1}, A_{2}\right)$ and $\left(B_{1}, B_{2}\right)$ are synchronous, by Lemma 3 we have $\left.\left(A_{1}-B_{1}\right)\right)\left(A_{2}-B_{2}\right)$ Ö 0 . By using Lemma 3 again, we obtain

$$
\begin{aligned}
& \left.0 \tilde{\mathrm{N}} t(1-t)\left[\left(A_{1}-B_{1}\right)\right)\left(A_{2}-B_{2}\right)\right]
\end{aligned}
$$

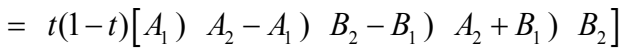

$$
\begin{aligned}
& =\left[\begin{array}{ll}
(1-t)\left(A_{1}\right) & \left.\left.A_{2}\right)+t\left(B_{1}\right) B_{2}\right)
\end{array}\right] \\
& \text {-[(1-t) } \left.\left.A_{1}+t B_{1}\right]\right)\left[(1-t) A_{2}+t B_{2}\right] \\
& =\left[\begin{array}{lll}
\left(A_{1}\right) & \left.A_{2}\right) "{ }_{t}\left(B_{1}\right) & \left.B_{2}\right)
\end{array}\right]-\left[\begin{array}{lll}
\left.\left(A_{1} "{ }_{t} B_{1}\right)\right) & \left(A_{2}{ }^{\prime \prime} B_{2}\right)
\end{array}\right] .
\end{aligned}
$$

Thus $\left.\left.\left.\left(A_{1}{ }^{\prime \prime} B_{1}\right)\right)\left(A_{2}{ }^{\prime}{ }_{t} B_{2}\right) \tilde{\mathrm{N}}\left(A_{1}\right) A_{2}\right){ }^{\prime}{ }_{t}\left(B_{1}\right) B_{2}\right)$.

(ii) For the opposite synchronous case, we have $\left.\left(A_{1}-B_{1}\right)\right)\left(A_{2}-B_{2}\right) \tilde{\mathrm{N}} 0$ and hence the inequality (18) holds.

Corollary 10. If two Hermitian operators $A$ and $B$ are comparable, then for any $t \in[0,1]$,

$$
\begin{aligned}
& \left.\left.\left.\left(A "{ }_{t} B\right)\right)\left(A "{ }_{t} B\right) \mathrm{N}(A) A\right) "{ }_{t}(B) B\right), \\
& \left.\left.\left.\left(A "{ }_{t} B\right)\right)\left(B "{ }_{t} A\right) \text { Ö }(A) B\right) "{ }_{t}(B) A\right), \\
& \left.\left.\left.\left(A "{ }_{t} B\right)\right)\left(B^{-1}{ }_{t} A^{-1}\right) \tilde{\mathrm{N}}(A) B^{-1}\right) "{ }_{t}(B) A^{-1}\right), \\
& \left.\left.\left.\left(A^{\prime \prime}{ }_{t} B\right)\right)\left(A^{-1} "{ }_{t} B^{-1}\right) \text { Ö }(A) A^{-1}\right) "{ }_{t}(B) B^{-1}\right) .
\end{aligned}
$$

Here, in (21) and (22), we assume further that $A$ and $B$ are invertible.

Proof. The proof is similar to that of Corollary 8 .

\section{OPERATOR INEQUALITIES ON WEIGHTED CLASSICAL PYTHAGOREAN MEANS AND KHATRI-RAO PRODUCTS}

In this section, we present a number of operator inequalities involving Khatri-Rao products and weighted arithmetic/harmonic/ geometric means under suitable assumptions about synchronization, comparability, and spectra of operators.

Corollary 11. Let $A, B \in \mathrm{B}(\mathrm{H})^{+}$be comparable operators. If $A^{*} B=$ $B * A$, then for any $t \in[0,1]$,

$A * B \ddot{O}\left(A !_{t} B\right) *\left(A !_{1-t} B\right)$.

Proof. By making use of Lemma 5, property (3), Corollary 10 and property (2), we obtain

$$
\begin{aligned}
& A^{*} B=\left(A^{*} B\right) !_{t}(B * A) \\
& =\left[\begin{array}{ll}
Z^{*}(A) & B) Z
\end{array}\right] !_{t}\left[\begin{array}{ll}
Z^{*}(B) & A) Z
\end{array}\right] \\
& \text { Ö } \left.\left.Z^{*}[(A) B) !_{t}(B) A\right)\right] Z \\
& \text { Ö } \left.Z^{*}\left[\left(A !_{t} B\right)\right)\left(B !_{t} A\right)\right] Z \\
& =\left(A !_{t} B\right) *\left(B !_{t} A\right)=\left(A !_{t} B\right) *\left(A !_{1-t} B\right) .
\end{aligned}
$$

Theorem 12. Let $A, B \in \mathrm{B}(\mathrm{H})^{+}$and $t \in(0,1)$. If $A^{*} B=B^{*} A$, then $A * B \ddot{O}\left(A \#_{t} B\right) *\left(A \#_{1-t} B\right)$.
Proof. It follows Lemma 5, property (3), Proposition 1, and property (2) that

$$
\begin{aligned}
& A^{*} B=\left(A^{*} B\right) \#_{t}\left(B^{*} A\right) \\
& =\left[\begin{array}{ll}
Z^{*}(A) & B) Z
\end{array}\right] \#_{t}\left[\begin{array}{ll}
Z^{*}(B) & A) Z
\end{array}\right] \\
& \text { Ö } \left.Z^{*}\left[\begin{array}{lll}
(A) & B) \#_{t}(B) & A
\end{array}\right)\right] Z \\
& =Z^{*}\left[\begin{array}{ll}
\left.\left(A \#_{t} B\right)\right) & \left.\left(B \#_{t} A\right)\right] Z
\end{array}\right. \\
& =\left(A \#_{t} B\right) *\left(B \#_{t} A\right) \\
& =\left(A \#_{t} B\right) *\left(A \#_{1-t} B\right) \text {. }
\end{aligned}
$$

Corollary 13. For any $X, Y \in \mathrm{B}(\mathrm{H})^{+}$such that $Y$ is invertible, we have

$$
Y^{*}\left(X Y^{-1} X\right) \text { Ö } X^{*} X .
$$

Proof. Note that for any $A, B \in \mathrm{B}(\mathrm{H})^{+}$such that $A$ is invertible, we have $A \#\left(B A^{-1} B\right)=B$. It follows this fact and Theorem 12 with $t=1 / 2$ that

$$
Y^{*}\left(X Y^{-1} X\right) \text { Ö }\left(Y \# X Y^{-1} X\right) *\left(Y \# X Y^{-1} X\right)=X^{*} X .
$$

Theorem 14. Let $A, B \in \mathrm{B}(\mathrm{H})^{+}$and $t \in(0,1)$. If $A^{*} B=B * A$, then $A^{*} B$ Ö $\left(A "{ }_{t} B\right) *\left(A !_{1-t} B\right)$.

Proof. Recall that the Khatri-Rao product is continuous with respect to the operator norm (see [9]). By continuity of the Khatri-Rao product, the $t$-weighted arithmetic mean and the $t$-weighted harmonic mean, we may assume that $A$ and $B$ are invertible.

$$
\left(X^{-1}+Y^{-1}\right)^{-1}=X-X(X+Y)^{-1} X \text {. }
$$

It follows this fact and Corollary 13 that

$$
\begin{aligned}
&\left(A^{\prime \prime}{ }_{t} B\right)^{*}\left(A !_{1-t} B\right) \\
&= {[(1-t) A+t B] *\left[t A^{-1}+(1-t) B^{-1}\right]^{-1} } \\
&= {[(1-t) A+t B]^{*} t^{-1}\left\{A-(1-t) A[(1-t) A+t B]^{-1} A\right\} } \\
&= {[(1-t) A+t B]^{*} t^{-1} A } \\
&-[(1-t) A+t B] *\left\{t^{-1}(1-t) A[(1-t) A+t B]^{-1} A\right\} \\
&= t^{-1}(1-t)\left(A^{*} A\right)+B^{*} A \\
&-t^{-1}(1-t)[(1-t) A+t B]^{*}\left\{A[(1-t) A+t B]^{-1} A\right\} \\
& \tilde{\mathrm{N}} t^{-1}(1-t)\left(A^{*} A\right)+A^{*} B-t^{-1}(1-t)\left(A^{*} A\right) \\
&= A^{*} B .
\end{aligned}
$$

In [8], it was shown that for any $A_{1}, B_{1}, A_{2}, B_{2}$ in $\mathrm{B}(\mathrm{H})^{+}$and $t \in[0,1]$,

$$
\left(A_{1} \#_{t} B_{1}\right) *\left(A_{2} \#_{t} B_{2}\right) \tilde{\mathrm{N}}\left(A_{1} * A_{2}\right) \#_{t}\left(B_{1} * B_{2}\right) .
$$

Now, we give a reverse inequality of (23). Recall that a linear map $\Phi$ between two operator algebras is said to be positive if it preserves positive operators; $\Phi$ is said to be unital if it preserves the identity operator.

Lemma 15 ([10]). Let $A, B \in \mathrm{B}(\mathrm{H})^{+}$be such that $m_{1} I \tilde{\mathrm{N}} A \tilde{\mathrm{N}} M_{1} I$ and $m_{2} I \tilde{\mathrm{N}} B \tilde{\mathrm{N}} M_{2} I$ where $m_{1}, m_{2}, M_{1}, M_{2}$ are positive constants. Denote $m=m_{2} / M_{1}$ and $M=M_{2} / m_{1}$. Then, for any positive linear map $\Phi: \mathrm{B}(\mathrm{H}) \rightarrow \mathrm{B}(\mathrm{H})$ and $t \in(0,1)$, we have

$$
\Phi\left(A \#_{t} B\right) \text { Ö } \lambda\left[\Phi(A) \#_{t} \Phi(B)\right]
$$




$$
\lambda=\frac{M m^{t}-m M^{t}}{(1-t)(M-m)} \cdot\left(\frac{1-t}{t} \cdot \frac{M^{t}-m^{t}}{M m^{t}-m M^{t}}\right)^{t} .
$$

Theorem 16. Let $A_{1}, B_{1}, A_{2}, B_{2} \in \mathrm{B}(\mathrm{H})^{++}$be such that $\left.0<m_{1} I \tilde{\mathrm{N}} A_{1}\right) \quad A_{2} \tilde{\mathrm{N}} M_{1} I$ and $\left.0<m_{2} I \tilde{\mathrm{N}} B_{1}\right) B_{2} \tilde{\mathrm{N}} M_{2} I$.

Let $m=m_{2} / M_{1}$ and $M=M_{2} / m_{1}$. Then for any $0<t<1$, we have

$$
\left(A_{1} \#_{t} B_{1}\right) *\left(A_{2} \#_{t} B_{2}\right) \text { Ö } \lambda\left[\left(A_{1} * A_{2}\right) \#_{t}\left(B_{1} * B_{2}\right)\right]
$$

where $\lambda$ is given by (25).

Proof. Consider a map $\Phi: T \quad Z^{*} T Z$ where $Z$ is the selection operator described in Lemma 5 , associated with the ordered tuple $(\mathrm{H}, \mathrm{H})$. Then, $\Phi$ is a unital positive linear map. It follows Lemma 5 , Proposition 1, and Lemma 15 that

$$
\begin{aligned}
& \left.\left(A_{1} \#_{t} B_{1}\right) *\left(A_{2} \#_{t} B_{2}\right)=Z^{*}\left[\left(A_{1} \#_{t} B_{1}\right)\right)\left(A_{2} \#_{t} B_{2}\right)\right] Z \\
& =Z^{*}\left[\left(\begin{array}{ll}
\left(A_{1}\right) & \left.\left.A_{2}\right) \#_{t}\left(B_{1}\right) B_{2}\right)
\end{array}\right] Z\right. \\
& \text { Ö } \lambda\left\{\left[\begin{array}{ll}
Z^{*}\left(A_{1}\right) & \left.A_{2}\right) Z
\end{array}\right] \#_{t}\left[\begin{array}{ll}
Z^{*}\left(B_{1}\right) & \left.B_{2}\right) Z
\end{array}\right]\right\} \\
& =\lambda\left[\left(A_{1} * A_{2}\right) \#_{t}\left(B_{1} * B_{2}\right)\right] \text {. }
\end{aligned}
$$

Corollary 17. Let $A, B \in \mathrm{B}(\mathrm{H})^{++}$and $t \in(0,1)$. Let $m_{1}, m_{2}, M_{1}, M_{2}$ be positive constants, denote $m=m_{2} / M_{1}$ and $M=M_{2} / m_{1}$, and define $\lambda$ as in (25).

(i) If $\left.m_{1} I \tilde{\mathrm{N}} A\right) A \tilde{\mathrm{N}} M_{1} I$ and $\left.m_{2} I \tilde{\mathrm{N}} B\right) B \tilde{\mathrm{N}} M_{2} I$, then $\left(A \#_{t} B\right) *\left(A \#_{t} B\right)$ Ö $\lambda\left[(A * A) \#_{t}(B * B)\right]$.

(ii) If $\left.m_{1} I \tilde{\mathrm{N}} A\right) \quad B \tilde{\mathrm{N}} M_{1} I$ and $\left.m_{2} I \tilde{\mathrm{N}} B\right) A \tilde{\mathrm{N}} M_{2} I$, then $\left(A \#_{t} B\right)^{*}\left(A \#_{1-t} B\right)$ Ö $\lambda\left[\left(A^{*} B\right) \#_{t}\left(B^{*} A\right)\right]$.

Proof The assertion (i) follows from Theorem 16 by setting $A_{1}=A_{2}:=A$ and $B_{1}=B_{2}:=B$. To prove (ii), set $A_{1}=B_{2}:=A$ and $B_{1}=A_{2}:=B$ in the same theorem and property (2).

Recall the following relation between a positive linear map and the harmonic mean of operators:

Lemma 18 ([10]). Let $A, B \in \mathrm{B}(\mathrm{H})^{++}$be such that $0<m_{1} I \tilde{\mathrm{N}} A \tilde{\mathrm{N}} M_{1} I$ and $0<m_{2} I \tilde{\mathrm{N}} B \tilde{\mathrm{N}} M_{2} I$. If $\Phi: \mathrm{B}(\mathrm{H}) \rightarrow \mathrm{B}(\mathrm{H})$ is a positive linear map, then

$$
\Phi(A ! B) \text { Ö } \frac{\left(\sqrt{M_{1} m_{1}}+\sqrt{M_{2} m_{2}}\right)^{2}}{\left(M_{1}+m_{2}\right)\left(m_{1}+M_{2}\right)}[\Phi(A) ! \Phi(B)] .
$$

Theorem 19. Let $A_{1}, B_{1}, A_{2}, B_{2} \in \mathrm{B}(\mathrm{H})^{++}$and $t \in[0,1]$.

(i) Suppose $\left.0<m_{1} I \tilde{\mathrm{N}} A_{1}\right) \quad A_{2} \tilde{\mathrm{N}} M_{1} I$ and $\left.0<m_{2} I \tilde{\mathrm{N}} B_{1}\right) \quad B_{2} \tilde{\mathrm{N}} M_{2} I$.If $\left(A_{1}, A_{2}\right)$ and $\left(B_{1}, B_{2}\right)$ are synchronous, then

$$
\left(A_{1} !_{t} B_{1}\right) *\left(A_{2} !_{t} B_{2}\right) \text { Ö } k^{2}\left[\left(A_{1} * A_{2}\right) !_{t}\left(B_{1} * B_{2}\right)\right]
$$

where

$$
k=\frac{\left(m_{1} \# M_{1}\right){ }_{1-t}\left(m_{2} \# M_{2}\right)}{\left(m_{1}{ }_{1-t} M_{2}\right) \#\left(M_{1}{ }_{1-t} m_{2}\right)} \text {. }
$$

(ii) If $\left(A_{1}, A_{2}\right)$ and $\left(B_{1}, B_{2}\right)$ are opposite synchronous, then

$$
\left(A_{1} !_{t} B_{1}\right) *\left(A_{2} !_{t} B_{2}\right) \tilde{\mathrm{N}}\left(A_{1} * A_{2}\right) !_{t}\left(B_{1} * B_{2}\right) \text {. }
$$

Proof. (i) Assume that $\left(A_{1}, A_{2}\right)$ and $\left(B_{1}, B_{2}\right)$ are synchronous. The cases $t=0$ and $t=1$ are trivial. Now, $t \in(0,1)$. By using Lemmas 5 and 18 , and Theorem 12, we have

$$
\begin{aligned}
& \left(A_{1} !_{t} B_{1}\right) *\left(A_{2} !_{t} B_{2}\right) \\
& \left.\quad=Z^{*}\left[\left(A_{1} !_{t} B_{1}\right)\right)\left(A_{2} !_{t} B_{2}\right)\right] Z
\end{aligned}
$$

$$
\begin{aligned}
& \text { Ö } Z^{*}\left[\left(\begin{array}{ll}
\left.A_{1}\right) & \left.\left.A_{2}\right) !_{t}\left(B_{1}\right) B_{2}\right)
\end{array}\right] Z\right. \\
& =Z^{*}\left\{\left[\begin{array}{ll}
\frac{1}{1-t}\left(A_{1}\right) & \left.A_{2}\right)
\end{array}\right] !\left[\begin{array}{ll}
\frac{1}{t}\left(B_{1}\right) & \left.B_{2}\right)
\end{array}\right]\right\} Z \\
& \text { Ö } \frac{\left(\frac{\sqrt{M_{1} m_{1}}}{1-t}+\frac{\sqrt{M_{2} m_{2}}}{t}\right)^{2}}{\left(\frac{M_{1}}{1-t}+\frac{m_{2}}{t}\right)\left(\frac{m_{1}}{1-t}+\frac{M_{2}}{t}\right)} \\
& \left.\quad \times\left\{\left[\frac{1}{1-t} Z^{*}\left(A_{1}\right) A_{2}\right) Z\right] !\left[\begin{array}{ll}
\frac{1}{-} Z^{*}\left(B_{1}\right) & \left.B_{2}\right) Z
\end{array}\right]\right\} \\
& =\left(\frac{\left(m_{1} \# M_{1}\right){ }_{1-t}\left(m_{2} \# M_{2}\right)}{\left(M_{1}{ }^{\prime}{ }_{1-t} m_{2}\right) \#\left(m_{1}{ }^{\prime}{ }_{1-t} M_{2}\right)}\right)^{2}\left[\left(A_{1} * A_{2}\right) !_{t}\left(B_{1} * B_{2}\right)\right] .
\end{aligned}
$$

Assume that $\left(A_{1}, A_{2}\right)$ and $\left(B_{1}, B_{2}\right)$ are opposite synchronous. By applying Lemma 5, Proposition 7 and property (3), we obtain that for any $t \in[0,1]$,

$$
\begin{aligned}
\left(A_{1} !_{t} B_{1}\right) *\left(A_{2} !_{t} B_{2}\right) & \left.=Z^{*}\left[\left(A_{1} !_{t} B_{1}\right)\right)\left(A_{2} !_{t} B_{2}\right)\right] Z \\
\tilde{\mathrm{N}} & Z^{*}\left[\begin{array}{lll}
\left(A_{1}\right) & \left.A_{2}\right) !_{t}\left(B_{1}\right) & \left.B_{2}\right)
\end{array}\right] Z
\end{aligned}
$$

$$
\begin{aligned}
& \left.\tilde{\mathrm{N}}\left[\begin{array}{ll}
Z^{*}\left(A_{1}\right) & \left.A_{2}\right) Z
\end{array}\right] !\left[Z^{*}\left(B_{1}\right) B_{2}\right) Z\right] \\
& \tilde{\mathrm{N}}\left(A_{1} * A_{2}\right) !_{t}\left(B_{1}^{*} B_{2}\right) .
\end{aligned}
$$

Corollary 20. Let $A, B \in \mathrm{B}(\mathrm{H})^{++}$and $t \in[0,1]$. Assume that $A$ and $B$ are comparable. Then

$$
\begin{aligned}
& \left(A !_{t} B\right) *\left(B !_{t} A\right) \tilde{\mathrm{N}}\left(A^{*} B\right) !_{t}\left(B^{*} A\right), \\
& \left(A !_{t} B\right) *\left(A^{-1} !_{t} B^{-1}\right) \tilde{\mathrm{N}}\left(A^{*} A^{-1}\right) !_{t}\left(B^{*} B^{-1}\right) .
\end{aligned}
$$

Moreover, if $0<\sqrt{m_{1}} I \tilde{\mathrm{N}} A \tilde{\mathrm{N}} \sqrt{M_{1}} I$ and $0<\sqrt{m_{2}} I \tilde{\mathrm{N}} B \tilde{\mathrm{N}} \sqrt{M_{2}} I$, then

$$
\left(A !_{t} B\right) *\left(A !_{t} B\right) \text { Ö } k^{2}\left[(A * A) !_{t}(B * B)\right] \text {. }
$$

Here, the constant $k$ is given by (28).

Proof. The results in this corollary are consequences of Theorem 4.9. The opposite synchronization between $(A, B)$ and $(B, A)$ leads to $(30)$. The opposite synchronization between $\left(A, A^{-1}\right)$ and $\left(B, B^{-1}\right)$ implies (31). If $0<\sqrt{m_{1}} I \tilde{\mathrm{N}} A \tilde{\mathrm{N}} \sqrt{M_{1}} I$ and $0<\sqrt{m_{2}} I \tilde{\mathrm{N}} B \tilde{\mathrm{N}} \sqrt{M_{2}} I$, then by Lemma 3, we have $\left.m_{1} I \tilde{\mathrm{N}} A\right) A \tilde{\mathrm{N}} M_{1} I$ and $\left.m_{2} I \tilde{\mathrm{N}} B\right) B$ $\tilde{\mathrm{N}} M_{2} I$, and the inequality (32) follows.

Theorem 21. Let $A_{1}, B_{1}, A_{2}, B_{2} \in \mathrm{B}(\mathrm{H})$ be Hermitian and $t \in[0,1]$.

(i) If $\left(A_{1}, A_{2}\right)$ and $\left(B_{1}, B_{2}\right)$ are synchronous, then

$$
\left(A_{1} "{ }_{t} B_{1}\right) *\left(A_{2}{ }_{t} B_{2}\right) \tilde{\mathrm{N}}\left(A_{1} * A_{2}\right){ }{ }_{t}\left(B_{1} * B_{2}\right) \text {. }
$$

(ii) If $\left(A_{1}, A_{2}\right)$ and $\left(B_{1}, B_{2}\right)$ are opposite asynchronous, the

$$
\left(A_{1}{ }_{t} B_{1}\right) *\left(A_{2} "{ }_{t} B_{2}\right) \text { Ö }\left(A_{1} * A_{2}\right){ }^{\prime}{ }_{t}\left(B_{1} * B_{2}\right) \text {. }
$$

Proof. (i) By using Lemma 5 and Theorem 9, we obtain

$$
\begin{aligned}
\left(A_{1}{ }_{t} B_{1}\right) *\left(A_{2} "{ }_{t} B_{2}\right) & \left.=Z^{*}\left[\left(A_{1} "{ }_{t} B_{1}\right)\right)\left(A_{2} "{ }_{t} B_{2}\right)\right] Z \\
& \tilde{\mathrm{N}} Z^{*}\left[\begin{array}{lll}
\left(A_{1}\right) & \left.A_{2}\right){ }^{\prime \prime}{ }_{t}\left(B_{1}\right) & \left.B_{2}\right)
\end{array}\right] Z \\
& \left.\left.=(1-t) Z^{*}\left(A_{1}\right) A_{2}\right) Z+t Z^{*}\left(B_{1}\right) B_{2}\right) Z \\
& =(1-t)\left(A_{1} * A_{2}\right)+t\left(B_{1} * B_{2}\right) \\
& =\left(A_{1} * A_{2}\right) "{ }_{t}\left(B_{1} * B_{2}\right) .
\end{aligned}
$$

(ii) The proof is similar to that of (i).

Corollary 22. If two Hermitian operators $A$ and $B$ are comparable, then 


$$
\begin{aligned}
& \left(A^{\prime \prime}{ }_{t} B\right) *\left(A^{\prime \prime}{ }_{t} B\right) \tilde{\mathrm{N}}\left(A^{*} A\right){ }{ }_{t}\left(B^{*} B\right), \\
& \left(A^{\prime}{ }_{t} B\right) *\left(B^{\prime}{ }_{t} A\right) \text { Ö }\left(A^{*} B\right) "{ }_{t}\left(B^{*} A\right), \\
& \left(A^{\prime \prime}{ }_{t} B\right) *\left(B^{-1}{ }_{t} A^{-1}\right) \tilde{\mathrm{N}}\left(A^{*} B^{-1}\right){ }_{t}\left(B^{*} A^{-1}\right), \\
& \left(A^{\prime \prime}{ }_{t} B\right) *\left(A^{-1}{ }_{t} B^{-1}\right) \text { Ö }\left(A^{*} A^{-1}\right) "{ }_{t}\left(B^{*} B^{-1}\right) .
\end{aligned}
$$

Here, in (37) and (38), we assume further that $A$ and $B$ are invertible.

Proof. The proof is similar to that of Corollary 8 .

\section{CONCLUSIONS}

We provide a number of operator inequalities between three classical Pythagorean means and two kinds of operator products, namely, Tracy-Singh products and Khatri-Rao products. Each inequality is valid under certain assumptions relying on (opposite) synchronization, comparability, and spectra of operators. Our results include tensor product of operators, and Tracy-Singh and Khatri-Rao products of matrices as special cases.

\section{ACKNOWLEDGEMENT}

The first author expresses his gratitude towards Thailand Research Fund for providing the Royal Golden Jubilee PhD Scholarship, grant No. PHD60K0225 throughout his research.

\section{REFERENCES}

[1] Bhatia, R. Positive Definite Matrices, Princeton University Press, New Jersey, 2007.

[2] Hiai, F. 2010. Matrix analysis: matrix monotone functions, matrix means, and majorizations, Interdisciplinary Information Sciences. 16(2), 139248.

[3] Hiai, F. and Petz, D. Introduction to Matrix Analysis and Applications, Springer, New Delhi, 2014.

[4] Ploymukda, A. Chansangiam, P. and Lewkeeratiyutkul, W. 2018. Algebraic and order properties of Tracy-Singh products for operator matrices, Journal of Computational Analysis and Applications, 24(4), 656-664.

[5] Ploymukda, A. and Chansangiam, P. 2016. Khatri-Rao products for operator matrices acting on the direct sum of Hilbert spaces, Journal of Mathematics, Article ID 8301709, 7 pages. http://dx.doi.org/ 10.1155/2016/8301709.

[6] Ploymukda, A. and Chansangiam, P. 2017. Several Inequalities for Khatri-Rao Products of Hilbert Space Operators, Communications in Mathematics and Applications, 8(1), 45-60.

[7] Ploymukda, A. and Chansangiam, P. 2019. Khatri-Rao Products and Selection Operators, Journal of Computational Analysis and Applications, 27(2), 316-325.

[8] Ploymukda, A. and Chansangiam, 2018. P. Geometric means and TracySingh products for positive operators, Communications in Mathematics and Applications. 9(4), 475-488.

[9] Ploymukda, A. Chansangiam, P. and Lewkeeratiyutkul, W. 2018. Analytic properties of Tracy-Singh products for operator matrices, Journal of Computational Analysis and Applications, 24(4), 665-674.

[10] Mićić, J., Pečarić, J. and Seo, Y. 2000. Complementary inequalities to inequalities of Jensen and Ando based on the Mond-Pečarić method, Linear Algebra and its Applications. 318(1-3), 87-107. 